\title{
Physique de l'archéomagnétisme pour la datation de bâtiments du haut Moyen Âge
}

Philippe Lanos (philippe.lanos@univ-rennes1.fr)

Institut de recherche sur les archéomatériaux (IRAMAT-CRP2A, UMR CNRS 5060, Université Bordeaux-Montaigne) et Université Rennes 1

CRP2A, Maison de l'archéologie, Esplanade des Antilles, 33607 Pessac Cedex

\section{La physique}

de l'archéomagnétisme, basée

sur la mesure des aimantations

thermorémanentes et sur l'étude

du champ magnétique terrestre

dans le passé, permet de dater

les terres cuites archéologiques

restées en place depuis

leur dernière cuisson, mais aussi

celles qui ont été déplacées

depuis leur lieu de fabrication

jusqu'au lieu de la construction.

\section{La datation de monuments}

du haut Moyen Âge a permis

de nouvelles interprétations

des mesures d'aimantation

effectuées sur les briques mises

en œuvre dans les maçonneries.

Ces avancées récentes

sont illustrées ici dans le cas

de la tour-clocher de la collégiale

Saint-Martin à Angers.

\section{Principe}

\section{de l'archéomagnétisme}

L'archéomagnétisme (AM) est une méthode de datation fondée sur l'enregistrement des variations du champ magnétique terrestre (CMT) par les terres cuites archéologiques (fours, foyers, tuiles, briques, céramiques) [1]. Au cours du refroidissement consécutif à un chauffage à une température supérieure à la température de Curie des grains magnétiques présents dans l'argile (typiquement $700^{\circ} \mathrm{C}$ ), la terre cuite enregistre le CMT sous la forme d'une aimantation, dite thermorémanente (ATR), portée par ces grains. Cette ATR a la propriété d'être acquise parallèlement et proportionnellement au CMT ambiant. Sa mesure au laboratoire permet de remonter à la direction et à l'intensité du champ ancien qui l'a généré.

À la surface du globe, le vecteur CMT est caractérisé par trois composantes : l'inclinaison I, la déclinaison D (mesurées en degrés), et l'intensité F (mesurée en microteslas : $\mu \mathrm{T}$ ). L'inclinaison I est l'angle du vecteur par rapport au plan horizontal local. La déclinaison D est l'angle entre le Nord géographique et la projection sur le plan horizontal du vecteur CMT, dont la direction indique le Nord magnétique. Ces trois paramètres varient dans l'espace et dans le temps à différentes échelles.
Les variations lentes, à l'échelle de la décennie, du siècle et du millénaire, sont appelées "variations séculaires " et sont dues au noyau externe liquide et riche en fer de la Terre (voir encadré 1, p. 55). La variation passée du CMT peut être déterminée indirectement par l'analyse des ATR enregistrées par les terres cuites archéologiques et les roches restées en place depuis leur dernière cuisson.

La capacité d'une terre cuite à acquérir une ATR en présence d'un champ magnétique est due à la présence de minéraux ferro ou ferrimagnétiques (par exemple, la magnétite $\mathrm{Fe}_{3} \mathrm{O}_{4}$ ou l'hématite $\mathrm{Fe}_{2} \mathrm{O}_{3}$, de températures de Curie respectivement égales à $580^{\circ} \mathrm{C}$ et $675^{\circ} \mathrm{C}$ ), dispersés sous forme de grains fins dans la matrice argileuse. Les principes de cette acquisition ont été décrits par Louis Néel en 1955. L'ATR de la terre cuite est égale à la somme vectorielle des moments magnétiques rémanents des grains ferromagnétiques. En présence d'un champ magnétique de faible intensité $(<100 \mu \mathrm{T})$, cette ATR est parallèle et proportionnelle au CMT ambiant et constitue ainsi un enregistrement très stable au cours du temps (sur des millions d'années !) du CMT au moment du dernier refroidissement de la terre cuite.

L'étude de structures de référence datées par des méthodes indépendantes 


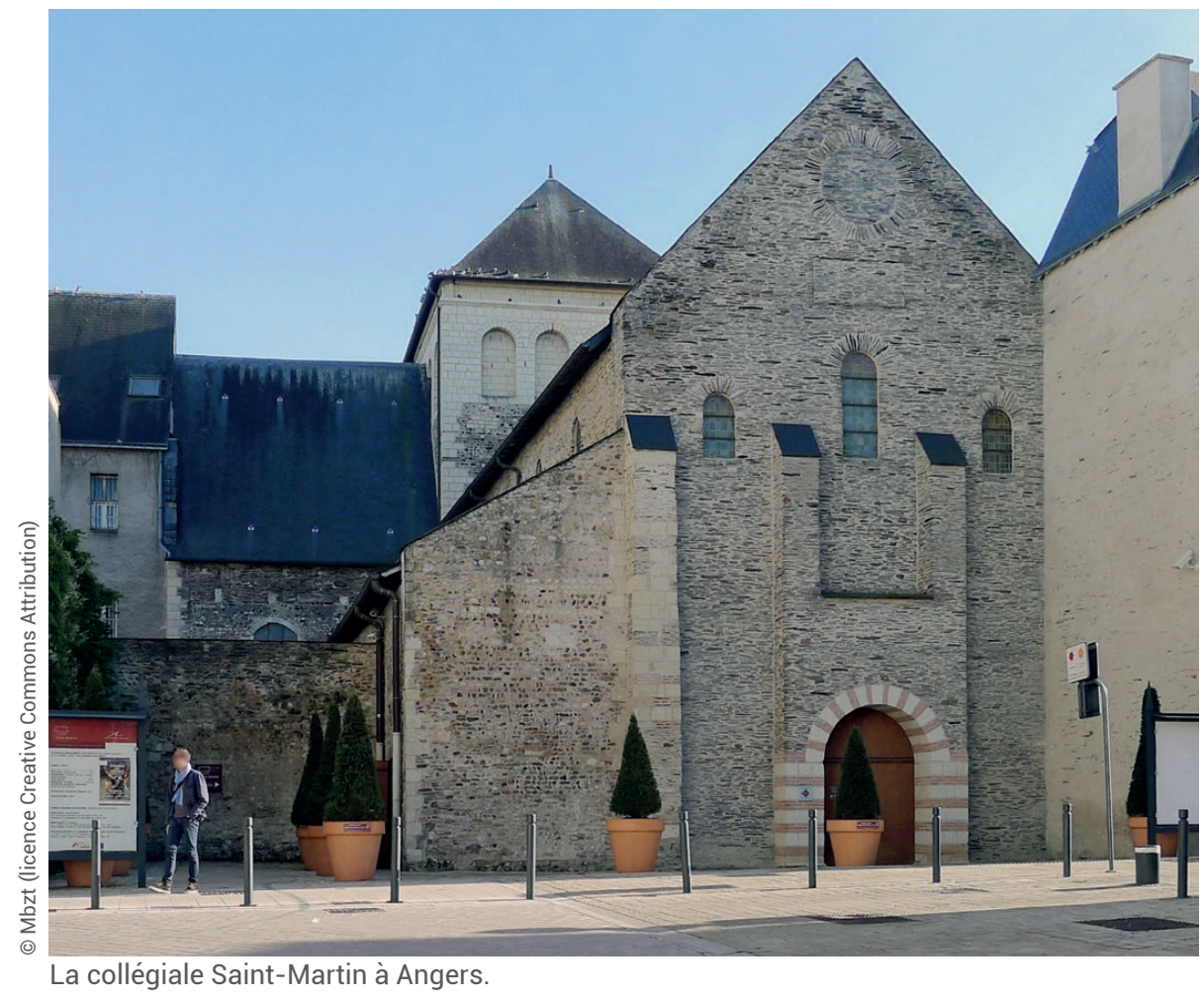

Variations du champ magnétique terrestre

Les premières observations du champ magnétique terrestre (CMT) datent de l'Antiquité, et la boussole apparait en Chine dès le $1^{\mathrm{er}}$ siècle de notre ère. L'hypothèse la plus simple pour expliquer le CMT, émise par Gilbert au $17^{\mathrm{e}}$ siècle, est d'assimiler le globe terrestre à un aimant permanent. Gellibrand observe en 1634 que la déclinaison magnétique varie dans le temps. C'est la première observation de la variation séculaire. Halley propose alors que le globe terrestre est une combinaison de plusieurs aimants en mouvement relatif les uns par rapport aux autres.

En 1838, Gauss donne la première décomposition mathématique du CMT en harmoniques sphériques et conclut qu'il est principalement dipolaire et d'origine interne. La découverte du point de Curie en 1895 démontre qu'au-dessus d'une certaine température, les matériaux sont désaimantés. La Terre possédant une chaleur centrale très importante, son centre ne peut pas être aimanté. Avec les avancées de la sismologie et la mise en évidence par Jeffreys, en 1926, que le noyau externe est liquide et métallique (il est composé principalement de fer), Elsasser propose en 1940 la théorie de la dynamo terrestre, ou géodynamo. Des questions restent en suspens concernant l'entretien de cette géodynamo. Une des hypothèses aujourd'hui est que la cristallisation du noyau solide interne (la " graine ") génère des courants de convection dans le noyau liquide, et que ces mouvements sont responsables de la dynamo terrestre.

Mais on a encore une idée imparfaite de la géométrie de ces mouvements et de leur évolution dans le temps, ainsi que de l'origine et de la fréquence des inversions du champ magnétique. Les géomagnéticiens ont développé trois approches pour comprendre les mouvements du fluide dans le noyau. La première repose sur l'étude de la variation spatiale et temporelle du CMT. Une seconde approche consiste à simuler numériquement l'écoulement du noyau et le champ magnétique engendré ; mais la difficulté reste dans l'ajustement des paramètres physiques. La troisième approche consiste à restituer les variations du CMT dans le passé à partir de l'étude de l'aimantation des roches (paléomagnétisme) et des terres cuites (archéomagnétisme). (histoire, archéologie, radiocarbone, luminescence, etc., voir encadré 2 p. 56) permet de construire des courbes de référence de variation séculaire de la direction (inclinaison, déclinaison) et de l'intensité du CMT en un lieu fixé (Paris pour la France). Ces courbes ne sont valables que dans une zone géographique réduite (1000 km de rayon au maximum), en raison de la variation spatiale du champ géomagnétique autour de sa forme dipolaire. Une fois établies, ces courbes peuvent servir à dater d'autres structures archéologiques dans la même aire géographique.

\section{Une méthode pour retrouver l'inclinaison du champ magnétique terrestre sur les terres cuites architecturales déplacées après leur cuisson}

Les travaux de recherche menés à Rennes dans les années 1980-90 ont permis d'étendre l'archéomagnétisme à la datation des terres cuites architecturales (TCA) grâce à la détermination de l'inclinaison, ceci malgré le fait que ces matériaux ont été bougés depuis leur dernière cuisson $[2,3,4]$. Nous 


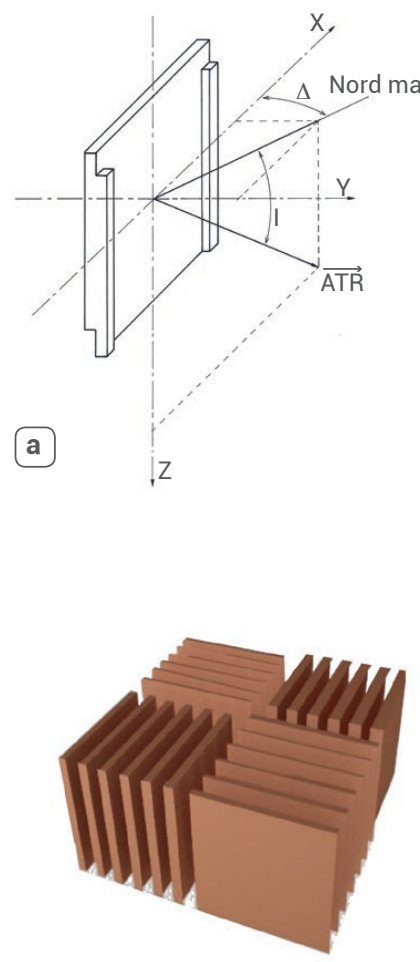

c

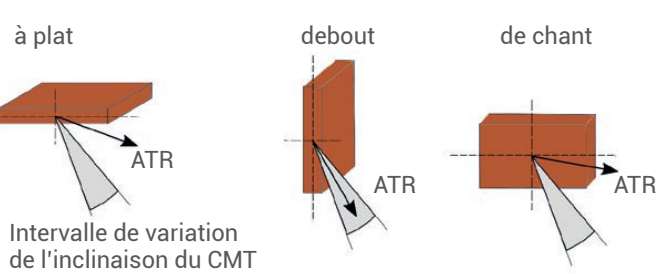

(b)

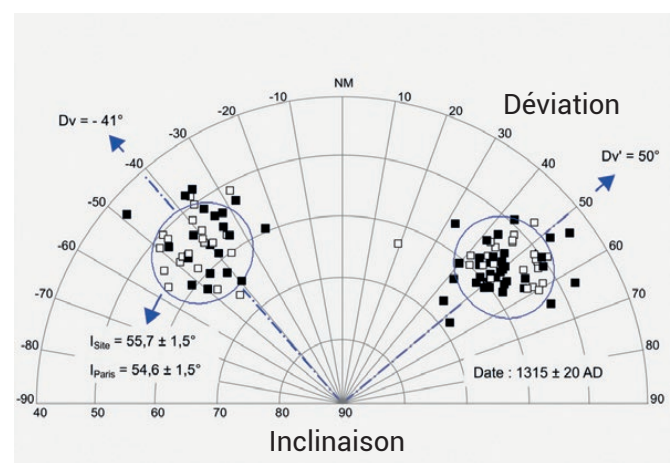

(d)
1. Analyse de l'aimantation d'un ensemble de briques cuites.

(a) Définition des angles inclinaison (I) et déviation $(\Delta)$ sur une tuile (ou brique) cuite sur tranche en position verticale.

(b) Test sur l'inclinaison permettant de restituer la position de cuisson (à plat, debout ou de chant) d'une brique dans l'hypothèse d'un chargement dans le four impliquant un axe $z$ selon la verticale.

(c) Schéma simplifié du mode de rangement des briques dans un four gallo-romain ou médiéval.

(d) Stéréogramme représentant l'inclinaison et la déviation des aimantations mesurées sur un ensemble de 106 briques médiévales cuites sur tranche en rangées perpendiculaires, provenant d'une fouille réalisée à Thérouanne (Pas-de-Calais) par H. Barbé, en 1993. La dispersion provient essentiellement des irrégularités du chargement. L'écart entre les déviations moyennes des deux groupes est proche de $90^{\circ}$, ce qui est une traduction directe du mode de rangement dans le four. C'est l'inclinaison moyenne déduite de ces deux groupes qui permet la datation (1315 \pm 20 apr. J.-C.)

Datations par radiocarbone et par thermoluminescence

La datation par radiocarbone consiste à mesurer le taux résiduel de l'isotope radioactif ${ }^{14} \mathrm{C}$ dans un organisme mort (si l'on considère une matière organique conservée sans échange avec l'environnement). Ce taux décroit au cours du temps selon une équation d'âge régie par la loi de désintégration radioactive du ${ }^{14} \mathrm{C}$ (qui se transmute en ${ }^{14} \mathrm{~N}$ par radioactivité bêta). Pour un taux résiduel mesuré, il est donc possible de déterminer le temps écoulé depuis la mort. On obtient ainsi un âge exprimé par rapport à l'année de référence 1950 (notée BP pour Before Present). La précision obtenue sur l'âge est de l'ordre de quelques pourcents.

La datation par thermoluminescence (voir l'article de P. Guibert, p. 8) repose sur la propriété de certains minéraux (quartz, feldspath, silex...) de stocker l'énergie provenant de la radioactivité naturelle (principalement des isotopes ${ }^{238} \mathrm{U},{ }^{232} \mathrm{Th}$ et ${ }^{40} \mathrm{~K}$ ) et de la restituer sous forme de lumière lorsqu'on les chauffe.

a) Les radioéléments, à l'état de traces, se désintègrent naturellement en émettant des rayonnements alpha (noyaux d'hélium), bêta (électrons) et gamma (ondes électromagnétiques de très courte longueur d'onde). Les rayons alpha ont une portée de 12 à $45 \mu \mathrm{m}$, les rayons bêta de 1 à $2 \mathrm{~cm}$ et les rayons gamma de 20 à $30 \mathrm{~cm}$.

b) Ces rayonnements excitent et déplacent les électrons des minéraux. Ceux-ci se piègent alors dans des sites cristallins jusqu'à ce qu'un apport d'énergie vienne les en libérer.

Le nombre d'électrons piégés, et par suite l'intensité de la lumière émise pendant le chauffage, sont d'autant plus grands que la quantité de rayonnement radioactif a été plus importante, donc le temps d'irradiation plus long. Pour un taux d'irradiation donné, le nombre d'électrons piégés sera proportionnel au temps écoulé depuis le début du processus. Une chauffe progressive à haute température, en laboratoire, provoque une émission de thermoluminescence (TL) qui est proportionnelle à l'énergie reçue par le minéral depuis la remise à zéro (chauffe archéologique). Ainsi, depuis sa cuisson, le minéral (quartz, feldspath...) reçoit une certaine dose d'énergie : c'est la dose archéologique (DA) qui est déterminée au laboratoire par mesure des émissions lumineuses.

L'âge de l'échantillon, c'est-à-dire le temps écoulé depuis le dernier chauffage qui a vidé les pièges (la cuisson d'une brique par exemple), est donné par le rapport entre la dose archéologique DA et la dose annuelle (aussi appelée débit de dose), mesurée par exemple sur le terrain à l'aide d'un dosimètre. La précision obtenue varie entre 5 et $10 \%$ de l'âge. 


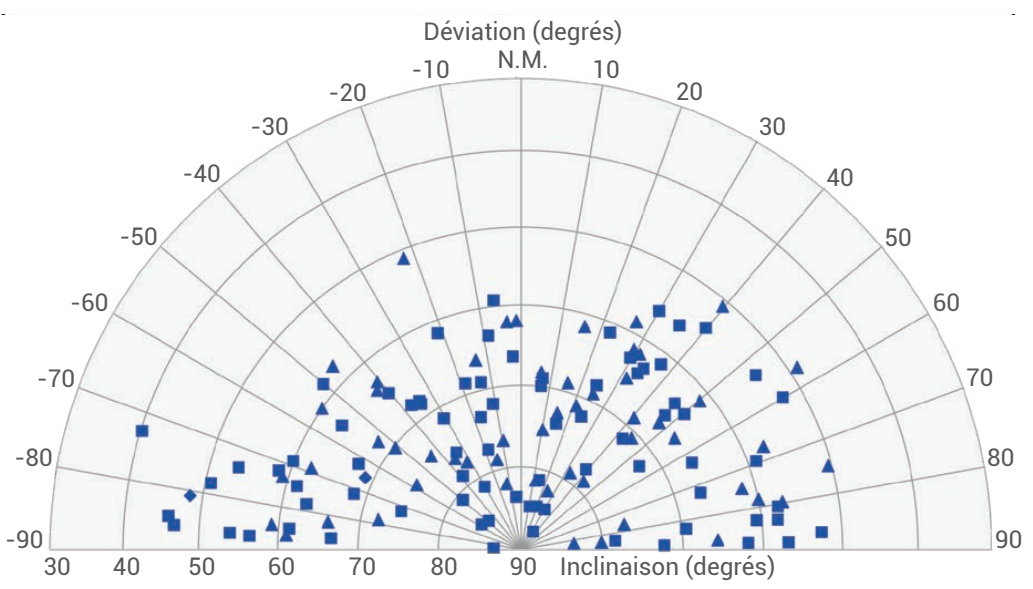

a
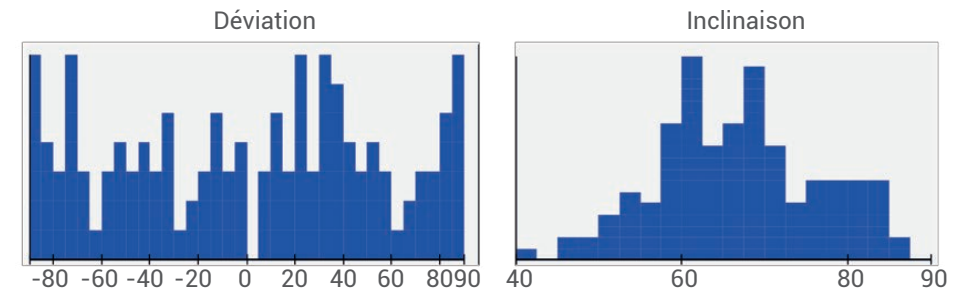

3. Directions des aimantations thermorémanentes mesurées.

(a) Stéréogramme (inclinaison en rayon - déviation en azimut) des aimantations ATR déterminées sur les briques prélevées. On observe une grande dispersion des directions, qui est due à l'affaissement de la charge lors de la cuisson.

(b) Histogrammes des déviations (de tendance uniforme entre $-90^{\circ}$ et $+90^{\circ}$ ) et des inclinaisons (de tendance gaussienne) déduits du stéréogramme. L'inclinaison moyenne et son écart-type permettent de retrouver l'inclinaison vraie ( $70 \pm 2$ degrés) du CMT lors de la cuisson.

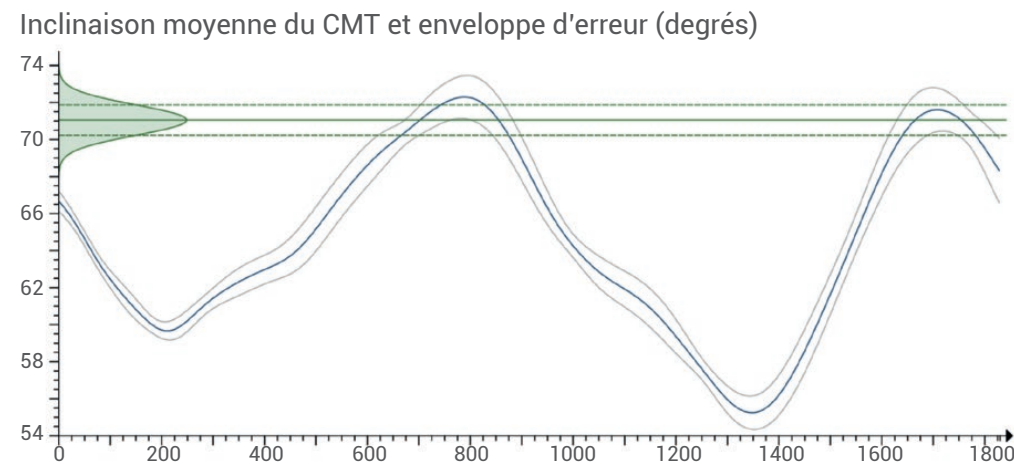

Distribution des dates de cuisson (à 95\% de confiance)

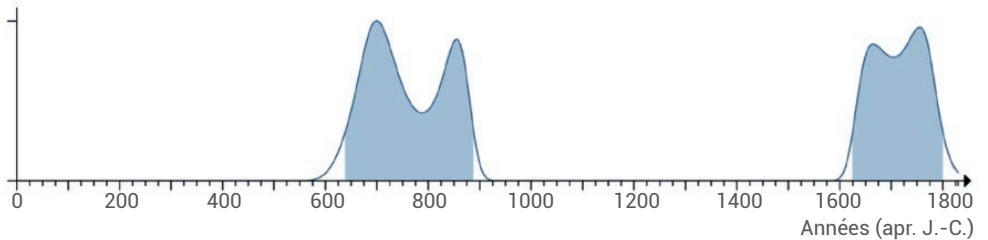

4. Datation archéomagnétique de la cuisson des briques de la collégiale Saint-Martin.

Le report de l'inclinaison moyenne mesurée à Angers et de son erreur (courbe gaussienne en vert en partie haute de la figure) sur la courbe de référence de la variation de l'inclinaison du CMT à Paris (représentée par sa moyenne en trait bleu et son enveloppe d'erreur à 95\%) permet de déterminer deux intervalles de date possible pour la cuisson des briques, au niveau de confiance de $95 \%$ : $[640 ; 890]$ AD à $53 \%$ de confiance et $[1625 ; 1800]$ AD à $42 \%$ de confiance (courbe de probabilité de date en bas de la figure, avec les deux intervalles figurés en bleu-gris).
$>>$

gramme (inclinaison I - déviation $\Delta$ ) en figure 3a. À notre surprise, nous avons constaté une très grande dispersion des directions d'aimantation qui montre que le mode de chargement des briques à la cuisson n'a plus rien à voir avec ceux observés classiquement pour l'époque gallo-romaine ainsi qu'à partir du $12^{\mathrm{e}}$ siècle (comparer la figure $3 a$ avec la figure 1d). L'histogramme des déviations observées est quasi uniforme tandis que l'histogramme des inclinaisons se rapproche d'une distribution gaussienne très étalée, avec une inclinaison moyenne de $66,7^{\circ}$ et un écart-type de $9,8^{\circ}$ (fig. 3b).

Pour expliquer cette dispersion, nous avons émis l'hypothèse que la position de cuisson sur tranche des briques s'était écartée aléatoirement et symétriquement par rapport à la verticale, suite à un affaissement de la charge dans le four. Ceci peut effectivement survenir lors d'une cuisson mixte chaux et briques, attestée par l'archéologie et par des observations récentes sur des ateliers en Tunisie. Les briques sont chargées audessus des pierres calcaires, en rangées croisées ou circulaires. Si l'on fait l'hypothèse d'un basculement aléatoire symétrique des briques par rapport à la verticale, les simulations numériques montrent qu'il est possible d'observer une dispersion très importante des ATR. Grâce à ces simulations, nous avons pu construire des abaques qui permettent de corriger l'inclinaison moyenne observée sur l'histogramme. Dans le cas d'Angers, l'inclinaison du CMT au site devient alors $70 \pm 2^{\circ}$, avec un angle de basculement moyen estimé à $11^{\circ}$. Ainsi, le basculement aléatoire de briques autour de la verticale peut conduire à une sous-évaluation notable de l'inclinaison vraie du CMT (ici un écart de plus de $3^{\circ}$ ).

\section{Datation archéomagnétique}

Pour procéder à la datation, l'inclinaison du CMT déterminée au site est ramenée à la valeur qu'elle aurait eu à Paris au même moment, grâce à une réduction géographique basée sur la forme dipolaire du CMT. On obtient ainsi la valeur de $71 \pm 2^{\circ}$. Le report de cette valeur sur la courbe de référence de l'inclinaison établie à Paris sur la période $[0 ; 1830]$ $\mathrm{AD}$ (fig. 4), fournit une distribution de dates, pour la fabrication des briques, caractérisée par deux intervalles : [640 ; $890] \mathrm{AD}$ à $53 \%$ et $[1625 ; 1800] \mathrm{AD}$ à $42 \%$ de niveau de confiance. Il est à 


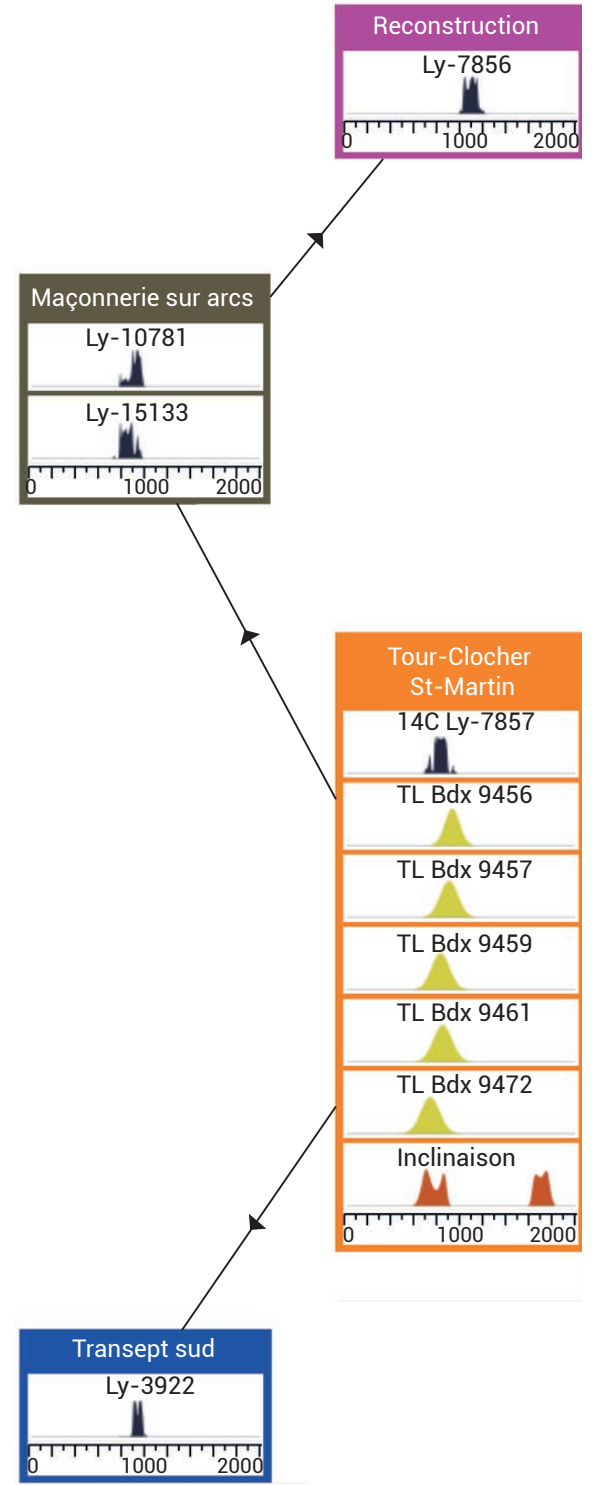

5. Modélisation chronologique de la tourclocher de la collégiale Saint-Martin à Angers. Modèle mis en place avec le logiciel ChronoModel à partir des informations chronologiques disponibles. Chacune des quatre boites correspond à un événement de construction et contient les distributions de dates calibrées. Les emplacements des prélèvements sont indiqués sur la figure $2 a$.

Dans le cas de l'évènement tour-clocher (couleur orange), les sept dates mesurées (une par ${ }^{14} \mathrm{C}$, cinq par thermoluminescence, une par archéomagnétisme) sont supposées contemporaines, aux erreurs près. Les trois autres évènements sont caractérisés chacun par une seule mesure au ${ }^{14} \mathrm{C}$. La datation relative du plus ancien vers le plus récent, représentée par les flèches, fournit une contrainte supplémentaire en imposant un ordre temporel aux quatre évènements. La combinaison de l'ensemble de ces informations fournit un intervalle de dates plus précis pour la construction de la partie "Tour-Clocher", soit entre 790 et $920 \mathrm{AD}$, à $95 \%$ de niveau de confiance. noter que la distribution de date dans le premier intervalle est bimodale, ce qui a son importance pour la modélisation chronologique qui suit.

\section{Modélisation chronologique pour préciser la datation de la tour-clocher}

Le programme d'étude sur la tourclocher comprenait, outre la datation archéomagnétique, une série de datations par radiocarbone $\left({ }^{14} \mathrm{C}\right)$ et par thermoluminescence (TL) opérées sur différents états identifiés dans la tour. Il était important de comparer ces trois datations en introduisant une information supplémentaire : la datation relative des différents états fournie par les observations d'archéologie du bâti. Pour la partie " tour-clocher " qui nous intéresse, nous disposons d'une datation au ${ }^{14} \mathrm{C}$ sur $\mathrm{du}$ charbon de bois retrouvé dans le mortier de maçonnerie, de cinq datations TL sur briques et de la datation AM par l'inclinaison. En supposant la contemporanéité de ces dates et en appliquant les contraintes de datation relative entre les différents états (fig. 5), nous avons pu calculer les distributions de date de chacun des états en utilisant le logiciel de modélisation chronologique ChronoModel (https://chronomodel.com/) [6]. Le calcul de la combinaison pour la tour-clocher montre alors que seul l'intervalle [640 ; 890] AD est compatible avec les autres datations, et mieux encore, que seule la partie autour du second mode de cet intervalle est cohérente avec les dates obtenues par radiocarbone et thermoluminescence. La modélisation chronologique permet ainsi de ne retenir qu'une seule des deux solutions chronologiques fournies par l'archéomagnétisme, et donc de resserrer la date de construction à l'intervalle [790;920] $\mathrm{AD}$, à 95\% de confiance.

\section{Conclusion}

En conclusion, l'analyse archéomagnétique a permis de mettre en évidence un mode original de positionnement des briques à la cuisson, différent de celui opéré à l'époque romaine et, plus tard, du Moyen Âge à nos jours. Nous avons montré qu'il était possible de corriger les effets de ce positionnement sur l'inclinaison moyenne des aimantations. Grâce à cette correction, la date fournie par l'archéomagnétisme devient cohérente avec les datations par radiocarbone et thermoluminescence. Enfin, la combinaison statistique de la datation archéomagnétique avec les autres datations montre que l'état " tour-clocher " de la collégiale Saint-Martin a été construit pratiquement un siècle et demi avant la date estimée jusque-là par les historiens de l'Art.

L'auteur remercie chaleureusement Didier Gourier pour la révision minutieuse du texte, ainsi que Philippe Dufresne pour la mise en forme des figures.

\section{Références}

1. Ph. Lanos, « L'archéomagnétisme », dans J. Evin et al., La datation en laboratoire (réédition 2005), pp. 124-170, collection «Archéologiques ", éditions Errance (Paris).

2. Ph. Lanos, "The effects of demagnetizing fields on thermoremanent magnetization acquired by parallel-sided baked clay blocks", Geophys.J. of R. Astr. Soc., 91 (1987) 985-1012.

3. Ph. Lanos, «Pratiques artisanales des briquetiers et archéomagnétisme des matériaux d'argile cuite. Une histoire de positions de cuisson ", Histoire \& Mesure, IX - 3/4 (1994) 287-304.
4-Ph. Lanos et al., "Archaeomagnetism, methodology and applications: implementation and practice of the archaeomagnetic method in France and Bulgaria", European Journal of Archaeology, 2 (1999) 365-392.

5. S. Blain et al., "Combined dating methods applied to building archaeology: the contribution of thermoluminescence to the case of the bell tower of St Martin's church, Angers (France)", Geochronometria, 38 (2011) 55-63.

6• Ph. Lanos et A. Philippe, "Hierarchical Bayesian modeling for combining dates in archaeological context", Journal de la SFdS 158 (2017) 72-88. 